\title{
Synthesis of 1-benzyloxypyrazin-2(1H)-one derivatives
}

\author{
Anh Hung Mai, Sonalika Pawar ${ }^{\dagger}$, Wim M. De Borggraeve* \\ Molecular Design and Synthesis, Chemistry Department, University of Leuven, Celestijnenlaan 200F-box 2404, 3001 Heverlee, Belgium
}

\section{A R T I C L E I N F O}

\section{Article history:}

Received 30 May 2014

Revised 23 June 2014

Accepted 25 June 2014

Available online 1 July 2014

\section{Keywords:}

Aspergillic acid

Hydroxamic acid

Pyrazin-2(1H)-one

Amidation

\begin{abstract}
A B S T R A C T
Different approaches for the synthesis of 1-benzyloxypyrazin-2(1H)-one derivatives from simple amino acids have been investigated. A library of 33 precursors for the preparation of $N$-hydroxy pyrazinones was obtained in moderate to good yields.
\end{abstract}

(c) 2014 Elsevier Ltd. All rights reserved.
The pyrazinone is a valuable scaffold in medicinal chemistry. It is present amongst others in the inhibition of HCV NS3 protease, ${ }^{1,2}$ neutrophil elastase, ${ }^{3}$ prolyl oligopeptidase, ${ }^{4}$ TF-FVIIa ${ }^{5}$ and thrombin. ${ }^{6,7}$ A lot of research in our group was directed towards the development of synthetic strategies for highly functionalized pyrazinones. ${ }^{8-15}$ Since there are bioactive compounds known in nature containing an $\mathrm{N}$-hydroxypyrazinone core (e.g., aspergillic acid, Fig. 1), ${ }^{16-18}$ we focused our attention to the development of new strategies to synthesize pyrazinones containing 1-benzyloxy functionality. These compounds can potentially give rise to aspergillic acid-like hydroxamic acids upon deprotection. Furthermore, in extension of our work on 1-alkyl/aryl functionalized pyrazinone3 -carboxamides, ${ }^{15}$ we also intended to prepare the corresponding 1-benzyloxy-3-carboxamide pyrazinones.

Our synthetic scheme for the synthesis of 1-benzyloxypyrazin$2(1 H)$-ones improves upon reported procedures ${ }^{19-23}$ and is similar to chemistry we already applied in the preparation of 1-alkyl/aryl pyrazinones. ${ }^{11,12}$ It relies on the base catalysed condensation of a glyoxal derivative with an amino acid hydroxamate. The condensations with phenyl glyoxal and diacetyl need a higher reaction temperature $\left(50-70^{\circ} \mathrm{C}\right)$ compared to those with glyoxal and methyl glyoxal. In order to avoid excessive side reactions resulting in very complex mixtures, it was important to use the glyoxal derivative as a limiting reagent ( 0.9 equiv, see Supplementary information) and to add it slowly to the reaction mixture via a syringe pump over the

\footnotetext{
* Corresponding author. Tel.: +32 16 327693; fax: +32 16327990.

E-mail address: wim.deborggraeve@chem.kuleuven.be (W.M. De Borggraeve).

† Current address: Chemistry Department, Fergusson College, F.C. Road, 411004 Pune, India.
}

course of 30-120 min. The regioselectivity of this reaction with an unsymmetrical glyoxal derivative (methyl/phenyl glyoxal) was reported before ${ }^{19,20}$ and was confirmed by us via NMR analysis. Proton H-6 in compounds $\mathbf{3 b}, \mathbf{c}, \mathbf{e}, \mathbf{f}, \mathbf{m}, \mathbf{n}, \mathbf{q}, \mathbf{t}, \mathbf{w}, \mathbf{y}, \mathbf{z c}, \mathbf{z g}$ (unsubstituted at position 6, but substituted at position 5) shows a clear NOE correlation with the methylene protons of the $O$-benzyl group, as well as an HMBC correlation with C-2 (Fig. 2).

The synthesis of 1-benzyloxy-3-alkylpyrazin-2(1H)-ones (3a-f, Scheme 1) was achieved by amidation of $N$-Boc protected amino acids 1 using $O$-benzyl hydroxylamine in combination with HOBt/EDCI/DIPEA/DMF, followed by Boc-deprotection and condensation. 0 -Benzyl hydroxamate 2 a could also be prepared via direct

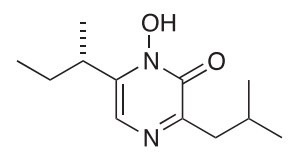

Figure 1. Aspergillic acid (1).

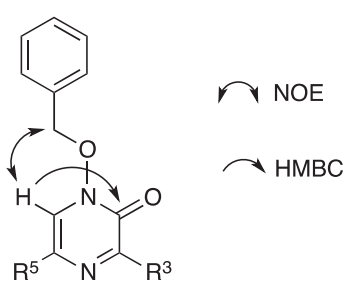

Figure 2. NOE and HMBC correlations of H-6 in compounds 3. 


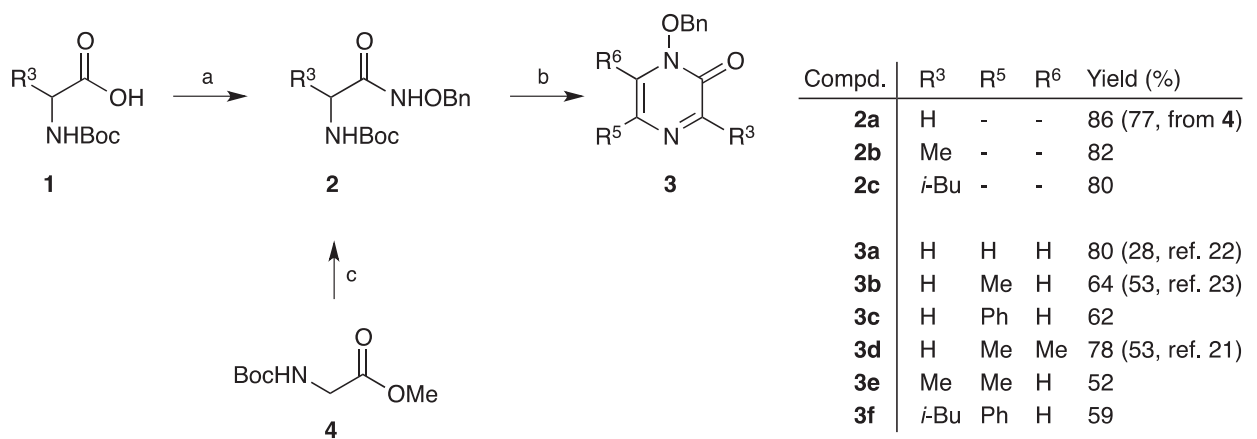

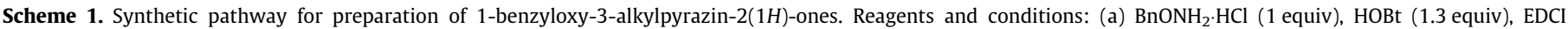

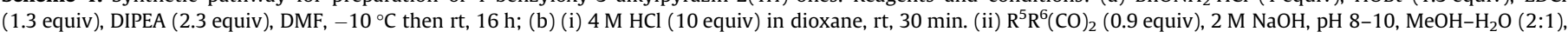
$-35^{\circ} \mathrm{C}$ then rt, overnight; (c) $\mathrm{BnONH}_{2}$ (1.1 equiv), LiHMDS (3.1 equiv), THF, $-78^{\circ} \mathrm{C}, 2 \mathrm{~h}$.

amidation of the methyl ester of amino acid $\mathbf{4}$ using LiHMDS base in THF. ${ }^{24} \mathrm{~A}$ slight reduction in yield and a shorter reaction time are observed in this instance (Scheme 1, entry 2a). The direct amidation route could also be applied to more complex substrates as exemplified below in Scheme 3. Using this approach of slow addition with glyoxal as the limiting reagent, the previously reported 1-benzyloxypyrazin-2(1H)-ones $\mathbf{3 a}$, $\mathbf{3 b}$ and $\mathbf{3 d}$ were obtained in better yields as compared to the literature. ${ }^{21-23}$

The synthesis of the novel and more complex 1-benzyloxypyrazin-2(1H)-one 3-carboxamides $\mathbf{3 g}-\mathbf{z g}$ is described in Schemes 2 and 3. In this pathway, the amino group in diethyl amino malonate ester hydrochloride (5) is first Boc-protected to form $\mathbf{6}$. This is followed by iterative mono-saponification amidation to generate $\mathbf{1 0}$ (Scheme 2). These compounds are then converted to pyrazine$2(1 \mathrm{H})$-ones $\mathbf{3 g}$ - $\mathbf{z g}$ in moderate to good yields after Boc-removal (Schemes 2 and 3). Compounds $\mathbf{1 0}$ can alternatively directly be obtained via conversion of ethyl ester 11 using LiHMDS and $\mathrm{NH}_{2} \mathrm{OBn}$ (Scheme 3). In the case of 3-carboxylated derivatives of 1-benzyloxypyrazin-2(1H)-one, the latter approach is able to reduce the number of reaction steps leading to the final products; however, it does limit the late stage diversification at C-3 of the target compounds, which in terms of library generation is a drawback.

In a further effort to shorten the synthetic protocol in the synthesis of 1-benzyloxypyrazin-2 $(1 H)$-ones $\mathbf{3}$, we performed the cyclization of the product of Boc-deprotection of $\mathbf{8}$ with glyoxals to generate 1-benzyloxypyrazin-2(1H)-one 3-carboxyl ethyl esters 12, which could be used as precursors in a one-step amidation to form 3 (Scheme 4) using $\mathrm{MgCl}_{2}$ as Lewis acid catalyst. ${ }^{25}$ The desired secondary amide products (3zc, 3zd and $\mathbf{3 z g}$ ) could be obtained in high yields by treating ethyl esters 12 with primary amines. However, no conversion was detected in case of secondary amines even after prolonged reaction time and heating at a temperature of $50{ }^{\circ} \mathrm{C}$ ( $\mathbf{3 z h}$ and $\mathbf{3 p}$ however can be synthesized via methods described in Schemes 2 and 3).

Using these approaches, a 33-component library of 1-benzyloxypyrazin-2 $(1 \mathrm{H})$-one derivatives, precursors for the synthesis of $\mathrm{N}$-hydroxypyrazinones, has been prepared in moderate to good

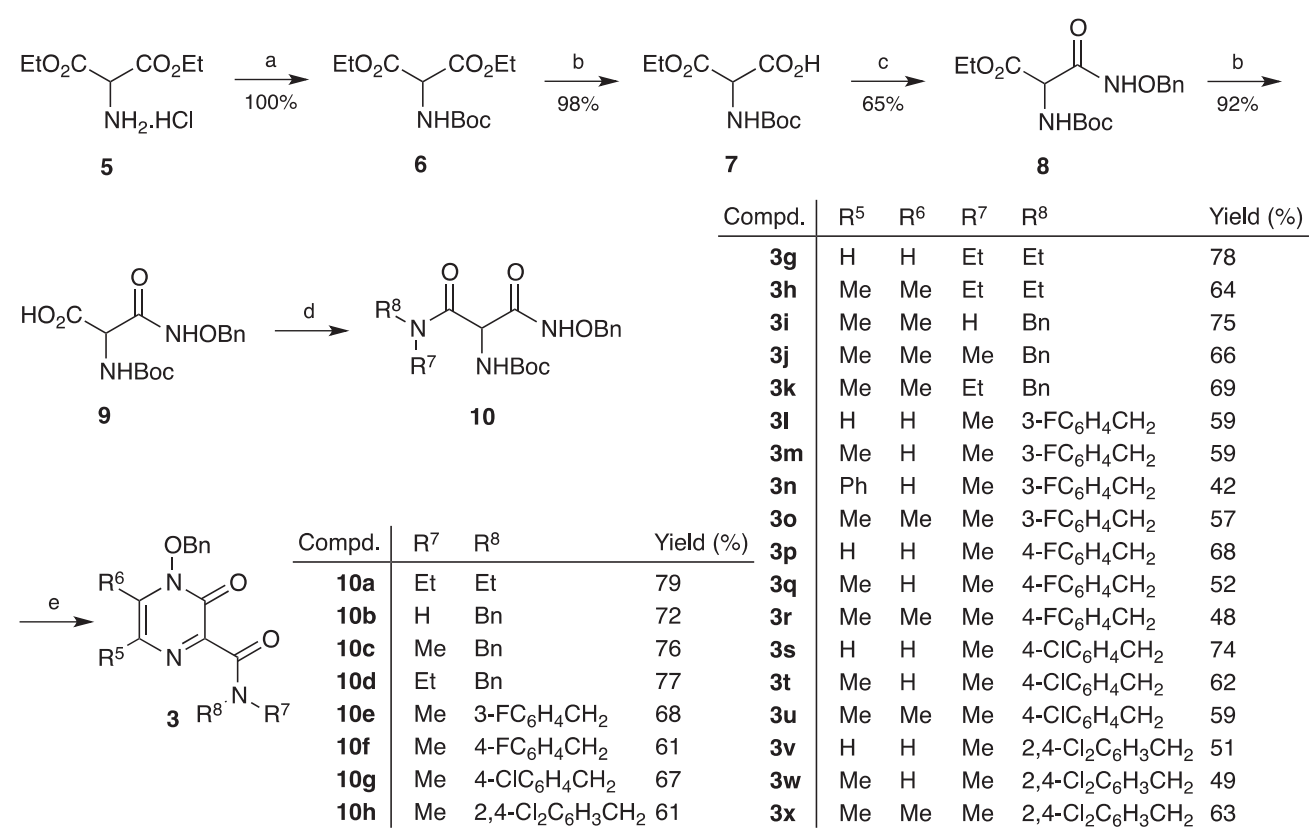

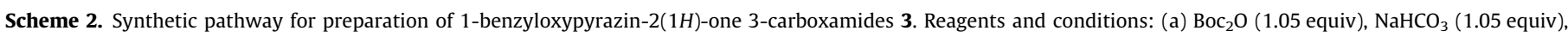

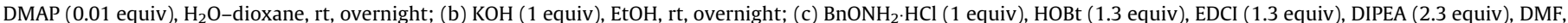

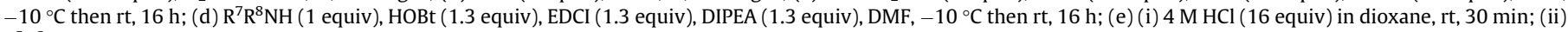
$\mathrm{R}^{5} \mathrm{R}^{6}(\mathrm{CO})_{2}$ (0.9 equiv), $2 \mathrm{M} \mathrm{NaOH}, \mathrm{pH} 8-10, \mathrm{MeOH}-\mathrm{H}_{2} \mathrm{O}(2: 1),-35^{\circ} \mathrm{C}$ then rt, overnight. 


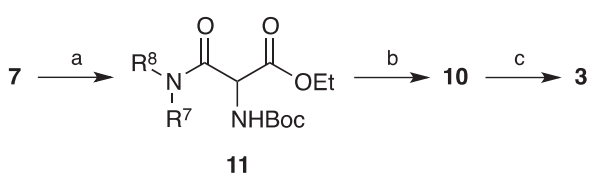

\begin{tabular}{r|lllll} 
Compd. & $\mathrm{R}^{5}$ & $\mathrm{R}^{6}$ & $\mathrm{R}^{7}$ & $\mathrm{R}^{8}$ & Yield (\%) \\
\hline 11a & - & - & $\mathrm{Et}$ & $\mathrm{Et}$ & 84 \\
11b & - & - & $\mathrm{H}$ & $3-\mathrm{FC}_{6} \mathrm{H}_{4} \mathrm{CH}_{2}$ & 73 \\
11c & - & - & $\mathrm{H}$ & $4-\mathrm{FC}_{6} \mathrm{H}_{4} \mathrm{CH}_{2}$ & 80 \\
11d & - & - & $\mathrm{H}$ & $4-\mathrm{ClC}_{6} \mathrm{H}_{4} \mathrm{CH}_{2}$ & 69 \\
11e & - & - & $\mathrm{H}$ & $3,4-\mathrm{Cl}_{2} \mathrm{C}_{6} \mathrm{H}_{3} \mathrm{CH}_{2}$ & 78 \\
& & & & & \\
10a & - & - & $\mathrm{Et}$ & $\mathrm{Et}$ & 79 \\
10i & - & - & $\mathrm{H}$ & $3-\mathrm{FC}_{6} \mathrm{H}_{4} \mathrm{CH}_{2}$ & 66 \\
10j & - & - & $\mathrm{H}$ & $4-\mathrm{FC}_{6} \mathrm{H}_{4} \mathrm{CH}_{2}$ & 64 \\
10k & - & - & $\mathrm{H}$ & $4-\mathrm{ClC}_{6} \mathrm{H}_{4} \mathrm{CH}_{2}$ & 57 \\
10I & - & - & $\mathrm{H}$ & $3,4-\mathrm{Cl}_{2} \mathrm{C}_{6} \mathrm{H}_{3} \mathrm{CH}_{2}$ & 67 \\
& & & & & \\
3y & $\mathrm{Me}$ & $\mathrm{H}$ & $\mathrm{Et}$ & $\mathrm{Et}^{2}$ & 42 \\
3z & $\mathrm{H}$ & $\mathrm{H}$ & $\mathrm{H}$ & $3-\mathrm{FC}_{6} \mathrm{H}_{4} \mathrm{CH}_{2}$ & 51 \\
3za & $\mathrm{Me}$ & $\mathrm{Me}$ & $\mathrm{H}$ & $3-\mathrm{FC}_{6} \mathrm{H}_{4} \mathrm{CH}_{2}$ & 47 \\
3zb & $\mathrm{H}$ & $\mathrm{H}$ & $\mathrm{H}$ & $4-\mathrm{FC}_{6} \mathrm{H}_{4} \mathrm{CH}_{2}$ & 60 \\
3zc & $\mathrm{Me}$ & $\mathrm{H}$ & $\mathrm{H}$ & $4-\mathrm{FC}_{6} \mathrm{H}_{4} \mathrm{CH}_{2}$ & 58 \\
3zd & $\mathrm{H}$ & $\mathrm{H}$ & $\mathrm{H}$ & $4-\mathrm{ClC}_{6} \mathrm{H}_{4} \mathrm{CH}_{2}$ & 67 \\
3ze & $\mathrm{Me}$ & $\mathrm{Me}$ & $\mathrm{H}$ & $4-\mathrm{ClC}_{6} \mathrm{H}_{4} \mathrm{CH}_{2}$ & 42 \\
3zf & $\mathrm{H}$ & $\mathrm{H}$ & $\mathrm{H}$ & $3,4-\mathrm{Cl}_{2} \mathrm{C}_{6} \mathrm{H}_{3} \mathrm{CH}_{2}$ & 61 \\
3zg & $\mathrm{Me}$ & $\mathrm{H}$ & $\mathrm{H}$ & $3,4-\mathrm{Cl}_{2} \mathrm{C}_{6} \mathrm{H}_{3} \mathrm{CH}_{2}$ & 49
\end{tabular}

Scheme 3. Direct amidation of ethyl ester 11. Reagents and conditions: (a) $R^{7} R^{8} N H$ (1 equiv), HOBt (1.3 equiv), EDCI (1.3 equiv), DIPEA (1.3 equiv), DMF, $-10^{\circ} \mathrm{C}$ then $\mathrm{rt}$, 16 h; (b) $\mathrm{BnONH}_{2}$ (1.1 equiv), LiHMDS (4.1 equiv), THF, $-78^{\circ} \mathrm{C}, 2 \mathrm{~h}$; (c) (i) $4 \mathrm{M} \mathrm{HCl}$ (16 equiv) in dioxane, rt, $30 \mathrm{~min}$. (ii) $\mathrm{R}^{5} \mathrm{R}^{6}(\mathrm{CO})_{2}(0.9$ equiv), $2 \mathrm{M} \mathrm{NaOH}, \mathrm{pH} \mathrm{8-10}$, $\mathrm{MeOH}-\mathrm{H}_{2} \mathrm{O}(2: 1),-35^{\circ} \mathrm{C}$ then rt, overnight.

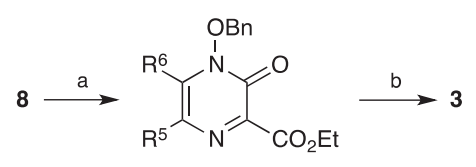

12

\begin{tabular}{r|lllll} 
Compd. & $\mathrm{R}^{5}$ & $\mathrm{R}^{6}$ & $\mathrm{R}^{7}$ & $\mathrm{R}^{8}$ & Yield (\%) \\
\hline 12a & $\mathrm{H}$ & $\mathrm{H}$ & - & - & 68 \\
12b & $\mathrm{Me}$ & $\mathrm{H}$ & - & - & 61 \\
& & & & & \\
3zc & $\mathrm{Me}$ & $\mathrm{H}$ & $\mathrm{H}$ & $4-\mathrm{FC}_{6} \mathrm{H}_{4} \mathrm{CH}_{2}$ & 95 \\
3zd & $\mathrm{H}$ & $\mathrm{H}$ & $\mathrm{H}$ & $4-\mathrm{ClC}_{6} \mathrm{H}_{4} \mathrm{CH}_{2}$ & 90 \\
3zg & $\mathrm{Me}$ & $\mathrm{H}$ & $\mathrm{H}$ & $3,4-\mathrm{Cl}_{2} \mathrm{C}_{6} \mathrm{H}_{3} \mathrm{CH}_{2}$ & 90 \\
3zh & $\mathrm{H}$ & $\mathrm{H}$ & $\mathrm{Me}$ & $\mathrm{Bn}$ & (c) \\
3p & $\mathrm{H}$ & $\mathrm{H}$ & $\mathrm{Me}$ & $4-\mathrm{FC}_{6} \mathrm{H}_{4} \mathrm{CH}_{2}$ & O(c,d)
\end{tabular}

Scheme 4. Synthesis of 3 via amidation of ester 12. Reagents and conditions: (a) (i) $4 \mathrm{M} \mathrm{HCl}$ (16 equiv) in dioxane, rt, $30 \mathrm{~min}$. (ii) $\mathrm{R}^{5} \mathrm{R}^{6}(\mathrm{CO})_{2}$ ( 0.9 equiv), $2 \mathrm{M} \mathrm{NaOH}, \mathrm{pH}$ 7-8, MeOH- $\mathrm{H}_{2} \mathrm{O}(2: 1),-35^{\circ} \mathrm{C}$ then rt, overnight; (b) (i) $\mathrm{MgCl}_{2}$ (2 equiv), THF, rt, 5 min. (ii) $R^{7} R^{8} \mathrm{NH}$ ( 2.5 equiv), rt, $16 \mathrm{~h}$; (c) No conversion, 12a was recovered (by LC-MS); (d) $38 \%$ yield of $\mathbf{3 p}$ (from $\mathbf{8}$, Scheme 2).

yields with minimal reaction steps. Pyrazin-2(1H)-ones 3c, 3e, $3 \mathbf{f}$ and 27 other 3-carboxamide substituted analogues $\mathbf{3 g}-\mathbf{z g}$ are new compounds. Deprotection of this library will be subject of another paper.

\section{Acknowledgment}

The authors gratefully acknowledge financial support from KU Leuven via OT/11/047 and the Ministry of Education and Training (Vietnam International Education Development, VIED).

\section{Supplementary data}

Supplementary data (general procedures for synthesis and characterization of all compounds, copies of ${ }^{1} \mathrm{H}$ and ${ }^{13} \mathrm{C}$ NMR spectra of compounds 3 and 12) associated with this article can be found, in the online version, at http://dx.doi.org/10.1016/j.tetlet. 2014.06.100. These data include MOL files and InChiKeys of the most important compounds described in this article.

\section{References and notes}

1. Örtqvist, P.; Gising, J.; Ehrenberg, A. E.; Vema, A.; Borg, A.; Karlén, A.; Larhed M. Danielson, U. H. Sandström, A Bioorg Med Chem. 2010, 18, 6512-6525.

2. Gising, J.; Belfrage, A. K.; Alogheli, H.; Ehrenberg, A.; Åkerblom, E.; Svensson, R.; Artursson, P.; Karlén, A.; Danielson, U. H.; Larhed, M.; Sandström, A. J. Med. Chem. 2013, 57, 1790-1801.

3. Hansen, P.; Ivarsson, M.; Lawitz, K.; Lönn, H.; Nikitidis, A.; Ray, A. U.S. Patent 8,114,881, Feb. 14, 2012.

4. Haffner, C. D.; Diaz, C. J.; Miller, A. B.; Reid, R. A.; Madauss, K. P.; Hassell, A. Hanlon, M. H.; Porter, D. J. T.; Becherer, J. D.; Carter, L. H. Bioorg. Med. Chem. Lett. 2008, 18, 4360-4363.

5. Zhang, X.; Glunz, P. W.; Jiang, W.; Schmitt, A.; Newman, M.; Barbera, F. A.; Bozarth, J. M.; Rendina, A. R.; Wei, A.; Wen, X.; Rossi, K. A.; Luettgen, J. M.; Wong, P. C.; Knabb, R. M.; Wexler, R. R.; Scott Priestley, E. Bioorg. Med. Chem. Lett. 2013, 23, 1604-1607.

6. Young, M. B.; Barrow, J. C.; Glass, K. L.; Lundell, G. F.; Newton, C. L.; Pellicore, J M.; Rittle, K. E.; Selnick, H. G.; Stauffer, K. J.; Vacca, J. P.; Williams, P. D.; Bohn, D.; Clayton, F. C.; Cook, J. J.; Krueger, J. A.; Kuo, L. C.; Lewis, S. D.; Lucas, B. J.; McMasters, D. R.; Miller-Stein, C.; Pietrak, B. L.; Wallace, A. A.; White, R. B.; Wong, B.; Yan, Y.; Nantermet, P. G. J. Med. Chem. 2004, 47, 2995-3008.

7. Kranjc, A.; Mašič, L. P.; Reven, S.; Mikic, K.; Preželj, A.; Stegnar, M.; Kikelj, D. Eur. J. Med. Chem. 2005, 40, 782-791.

8. Vekemans, J.; Pollers-Wieërs, C.; Hoornaert, G. J. Heterocycl. Chem. 1983, 20 919-923.

9. De Borggraeve, W. M.; Verbist, B. M. P.; Rombouts, F. J. R.; Pawar, V. G.; Smets, W. J.; Kamoune, L.; Alen, J.; Van der Eycken, E. V.; Compernolle, F.; Hoornaert, G. J. Tetrahedron 2004, 60, 11597-11612.

10. Heeres, J.; de Jonge, M. R.; Koymans, L. M. H.; Daeyaert, F. F. D.; Vinkers, M.; Van Aken, K. J. A.; Arnold, E.; Das, K.; Kilonda, A.; Hoornaert, G. J.; Compernolle, F.; Cegla, M.; Azzam, R. A.; Andries, K.; de Béthune, M.-P.; Azijn, H.; Pauwels, R.; Lewi, P. J.: Janssen, P. A. J. J. Med. Chem. 2004, 48, 1910-1918.

11. Azzam, R.; De Borggraeve, W.; Compernolle, F.; Hoornaert, G. J. Tetrahedron Lett. 2004, 45, 1885-1888.

12. Azzam, R.; De Borggraeve, W. M.; Compernolle, F.; Hoornaert, G. J. Tetrahedron 2005, 61, 3953-3962.

13. Pawar, V. G.; De Borggraeve, W. M. Synthesis 2006, 2006, 2799-2814.

14. Kamoune, L.; De Borggraeve, W. M.; Gielens, C.; Voet, A.; Robeyns, K.; De Maeyer, M.; Van Meervelt, L.; Compernolle, F.; Hoornaert, G. Eur. J. Org. Chem. 2007, 2007, 2977-2986.

15. Pawar, S. V.; Pawar, V. G.; Dehaen, W.; De Borggraeve, W. M. Org. Lett. 2008, 10, 4473-4476.

16. Dutcher, J. D. J. Biol. Chem. 1947, 171, 321-339.

17. Lott, W. A.; Shaw, E. J. Am. Chem. Soc. 1949, 71, 70-73.

18. Hogg, R. W.; Biswas, C. S.; Broquist, H. P. J. Bacteriol. 1965, 90, 1265-1270.

19. Dunn, G.; Ramsay, D. W. C.; Elvidge, J. A.; Spring, F. S.; Newbold, G. T.; Sweeny, W. Nature $1949,164,181$.

20. Jones, R. G. J. Am. Chem. Soc. 1949, 71, 78-81.

21. Katoh, A.; Ohkanda, J.; Itoh, Y.; Mitsuhashi, K. Chem. Lett. 1992, 21, 2009-2012.

22. Ohkanda, J.; Tokumitsu, T.; Mitsuhashi, K.; Katoh, A. Bull. Chem. Soc. Jpn. 1993, $66,841-847$

23. Ohkanda, J.; Kumasaka, T.; Takasu, A.; Hasegawa, T.; Katoh, A. Heterocycles 1996, 43, 883-889.

24. Gissot, A.; Volonterio, A.; Zanda, M. J. Org. Chem. 2005, 70, 6925-6928.

25. Guo, Z.; Dowdy, E. D.; Li, W.-S.; Polniaszek, R.; Delaney, E. Tetrahedron Lett. 2001, 42, 1843-1845. 